Relations industrielles

Industrial Relations

\title{
Lettre du Cardinal Tardini
}

Volume 15, numéro 4, octobre 1960

URI : https://id.erudit.org/iderudit/1021913ar

DOI : https://doi.org/10.7202/1021913ar

Aller au sommaire du numéro

Éditeur(s)

Département des relations industrielles de l’Université Laval

ISSN

0034-379X (imprimé)

1703-8138 (numérique)

Découvrir la revue

Citer ce document

(1960). Lettre du Cardinal Tardini. Relations industrielles / Industrial Relations, 15(4), 477-480. https://doi.org/10.7202/1021913ar

Tous droits réservés (C Département des relations industrielles de l’Université Laval, 1960
Ce document est protégé par la loi sur le droit d'auteur. L'utilisation des services d'Érudit (y compris la reproduction) est assujettie à sa politique d'utilisation que vous pouvez consulter en ligne.

https://apropos.erudit.org/fr/usagers/politique-dutilisation/ 


\section{INFORMATIONS}

\section{Syndicalisme et démocratisation de l'économie}

La Semaine Sociale du Canada (section française) a tenu ses assises annuelles en fin de septembre dans la ville de Trois-Rivières. Le suiet à l'étude, «Syndicalisme et organisation professionnelle», intéresse particulièrement nos lecteurs. Le texte complet des travaux qui ont été présentés paraîtra bientôt dans un livre. On trouvera ici un résumé des communications.

\section{LETTRE DU CARDINAL TARDINI}

Revenant sur un thème qui leur est devenu familier, les Semaines Sociales du Canada traiteront cette année du syndicalisme et de l'organisation professionnelle. Déjà en 1921, à Québec, elles avaient étudié le syndicalisme; en 1936, à Trois-Rivières, elles avaient abordé l'organisation professionnelle. Cette année, elles unissent ensemble ces thèmes, afin de montrer qu'elles ne considèrent pas le syndicalisme ou l'organisation professionnelle comme deux structures alternatives, l'une excluant l'autre, mais comme complémentaires: le syndicalisme appelle une organisation professionnelle, dans laquelle il vient s'intégrer; l'organisation professionnelle, à son tour, n'atteint pleinement son but que si elle s'appuie sur des unions librement constituées, où les différentes catégories sociales sont groupées suivant leurs affinités et leurs intérêts propres.

Un des points fondamentaux de l'enseignement de l'Eglise en cette matière,c'est le principe de la liberté syndicale. On l'a opposé à bon droit à la volonté arbitraire de l'Etat: peut-être a-t-on moins remarqué la variété de ses applications possibles. C'est aux membres de l'association, disait déjà Léon XIJI, qu'il appartient de «choisir librement la discipline et les lois qui leur paraissent les plus appropriées au but qu'ils poursuivent ». Ils le feront, ajoutait-il, en tenant compte «du génie de chaque nation, des essais tentés et de l'expérience acquise 》 (Rerum: Novarum (Acta Leonis XIII, XI, 1891, p. 138.) Cf. aussi Longinqua Oceani, (ibid, , XV, 1895, p. 17).

La liberté syndicale peut être mise en péril de plusieurs façons. Elle serait menacée, par exemple, si les organisations professionnelles devenaient un rouageadministratif ou politique de l'Etat, ou si, dotées de privilèges abusifs, certaines jouissaient d'un monopole juridique. Pie XI y faisait allusion à propos de la. «nouvelle organisation syndicale et corporative» alors appliquée en Italie. «On craint, observait-il,... qu'elle ne risque d'être mise au service de fins politiques particulières, plutôt que de contribuer à l'avènement d'un meilleur équilibre social 》 (Quadragesimo Anno, A.A.S. 23, 1931, p. 208).

Il est vrai que, depuis la dernière guerre mondiale, les syndicales, plus concentrées, sont devenues aussi plus indépendantes. Mais cette puissance accrue 
comporte à son tour un nouveau risque pour la liberté: que le syndicat en vienne un jour à «exercer une sorte de patronat ou de droit, en vertu duquel il disposerait librement du travailleur, de ses forces et de ses biens » (Pie XII, Allocution aux ACLI, 11 mars 1945, A.A.S. 37-1945, p. 70), ou qu'utilisant l'influence qu'il exerce naturellement sur la politique et sur l'opinion publique, il ne soit tenté d'abuser de la force que donne le nombre: tentation commune, du reste, aux syndicats patronaux et ouvriers, aux trusts économiques, à toutes ces forces collectives que constituent les différents groupements professionnels et sociaux. Ni la liberté ni la dignité ouvrière ne sont pleinement respectées quand «la défense des droits personnels du travailleur est de plus en plus aux mains d'une collectivité anonyme, qui agit par l'intermédiaire de gigantesques organisations tendant au monopole 》 (Pie XII, Radio-Message du 24 Décembre 1952, A.A.S. 45, 1953, p. 42).

Les travailleurs catholiques canadiens ne furent pas les derniers à entendre les enseignements des Souverains Pontifes en ces matières, et c'est bien légitimement qu'ils sont fiers d'une longue fidélité à la parole de l'Eglise. Ils ont choisi de donner leur préférence aux unions entre catholiques, parce qu'ils savent qu'il n'est pas d'action syndicale sans doctrine sociale; et ils se sont appliqués à faire de leurs unions, non seulement les organes de défense légitime des intérêts ouvriers, mais aussi des centres d'éducation et de formation doctrinale et morale, à laquelle leurs aumôniers ont largement participé. Ils ont de la sorte acquis une riche expérience, dont les leçons peuvent être opportunément utilisées aujourd'hui dans une conjoncture nouvelle. C'est ainsi, par exemple, que les unions syndicales entre catholiques pourraient fournir aux immigrants - attirés par l'expansion industrielle, mais bien souvent vulnérables, parce qu'ils sont comme on dit aujourd'hui, déracinés un appui moral auquel ne devraient pas mettre obstacle les différences de langue et d'origine. Cet accueil pourrait d'ailleurs s'étendre, si les circonstances le suggéraient, à tout travailleur qui accepterait pour base de son action syndicale les principes de la doctrine sociale enseignée par l'Eglise. De plus, bien loin de s'isoler, les unions catholiques de travailleurs canadiens ont l'ambition légitime d'apporter leur contribution constructive à la promotion ouvrière. Et dans cette perspective elles ne refuseraient pas certaines formes de collaboration avec les organisations syndicales qui ne s'excluent pas eiles-mêmes de cette coopération par la profession de doctrines subversives et négatrices de la foi chrétienne (Cf. Décret du S. Office, ler juillet 1949, A.A.S. 41, 1949, p. 334).

Bien des transformations sont survenues depuis l'époque de Léon XIII. Comme le signalait déjà Pie XI, les sages directives du grand pontife de l'encyclique Rerum Novarum furent appliquées de diverses manières suivant les temps et les lieux. Léon XIII assignait comme tâche aux associations de travailleurs, du moins ordinairement, à la fois la défense des intérêts matériels et la protection du bien religieux et moral des ouvriers, attribuant à ce dernier, comme il convenait, l'importance principale. A un demi-siècle de distance, Pie XII louait le Mouvement Ouvrier Chrétien belge d'avoir organisé des syndicats «qui s'efforcent de promouvoir Jordre chrétien dans le monde ouvrier», des coopératives chrétiennes qui ont contribué «à la sécurité du travailleur et de sa famille », des sociétés d'assurances mutuelles contre les accidents et la maladie, des institutions «destinées à la formation et à l'éducation des travailleurs 》 (Allocution du 11 septembre 1949, A.A.S. 41, 1949, p. 548). 
Ainsi une certaine «division du travail », suivant le mot de Pie XI, était intervenue. (Cf. Quadragesimo Anno, A.A.S. 23, 1931, p. 187).

Ailleurs il est arrivé que «soit la législation, soit certaines pratiques de la vie économique, soit la déplorable division des esprits et des coeurs (........) empéchaient de fonder des syndicats nettement catholiques. Dans de telles conjonctures, les ouvriers se voient pratiquement contraints de donner leur nom ì des syndicats neutres, où cependant on respecte la justice et l'équité, où pleine liberté est laissée aux fidèles d'obéir à la voix de l'Eglise »(Ibid).

Quand pareille nécessité pratique s'impose, on doit avoir soin, comme l'exigeait Saint Pie X et le rappelait Pie XI, que «toujours, à côté de ces syndicats, existent d'autres associations qui s'emploient à donner une sérieuse formation religieuse et morale (Enc. Singulari Quadam, 24 septembre 1912, A.A.S. 4, 1912, p. 660, et Quadragesimo Anno, i.c.).

Au reste, des Syndicats «neutres》 ou purement économiques n'échapperaient pas pour autant aux impératifs de la morale sociale, puisque celle-ci a son fondement dans la loi naturelle, dont l'Eglise - ici comme ailleurs - proclame et défend les exigences.

Il faut encore souligner une autre idée directrice qui court tout au long de l'enseignement pontifical, de Léon XIII à Jean XXIII. Pie XII l'exprimait en ces termes: « Au-dessus de la distinction entre employeurs et employés, les hommes doivent discerner et reconnaître l'unité supérieure qui lie entre eux tous ceux qui collaborent à la production, autrement dit leur solidarité dans la tâche de pourvoir ensemble et de façon stable au bien commun et aux besoins de toute la communauté (Allocution aux ACLI, 11 mars 1945, A.A.S. 37, 1945, p. 71).

De fait, les Souverains Pontifes ont toujours eu en vue, dans toute forme d'organisation professionnelle, non seulement la pacification des rapports sociaux, mais plus encore la constitution d'un ordre harmonieux où chacun, suivant sa condition, aurait sa part de responsabilités et sa participation équitable aux fruits de l'effort collectif. Ceci n'a peut-être pas été toujours suffisamment remarqué. Il ne s'agit pas seulement, en effet, d'une juste distribution de salaires, ni seulement de l'organisation interne de la profession à des fins économiques et sociales, mais bien plus encore d'un équilibre à trouver, d'une collaboration à organiser, d'initiatives à provoquer, de responsabilités à confier, à l'intérieur des corps professionnels, comme entre eux à l'intérieur de l'Etat, comme à l'intérieur de la communauté humaine.

Tous ces problèmes, anciens quant au fond, renouvelés quant à leurs incidences pratiques, vont être abordés par la prochaine Semaine Sociale du Canada. Elle y. apportera la lumière de la doctrine sociale chrétienne. Elle sait en effet qui \& ni l'organisation professionnelle et le syndicat, ni les commissions mixtes, ni le contrat collectif, ni l'arbitrage, ni toutes les prescriptions de la législation sociale la plus attentive et la plus avancée, ne parviennent à rétablir une concorde pleine et durable, à produire tous leurs fruits, si une action prévoyante et constante n'intervient pas, pour communiquer un souffle de vie spirituelle et morale à la constitution même des rapports économiques». (Allocution aux représentants des 
organisations patronales et ouvrières de l'industrie électrique italienne, 24 janvier 1946, Radio-Messagi e descorsi, T. VII, p. 350).

Sage avertissement de Pie XII, auquel fait écho l'enseignement du Pontife glorieusement régnant. Dès Sa première Encyclique, Sa Sainteté Jean XXIII demandait que «les droits et devoirs réciproques des employeurs et des travailleurs fussent mieux harmonisés et réglés ». Le Saint-Siège recommandait qu’on veillât « à ce qu'au progrèe économique... corresponde un non moindre progrès dans le domaine moral, comme le demande notre dignité de chrétiens et notre simple dignité d'hommes ». Et il concluait: «Ces perspectives se réaliseront le jour où la doctrine sociale de l'Eglise catholique sera mise pleinement en vigueur ». (Ad Petri Cathedram, A.A.S. 51, 1959, pp. 508-509).

Puisse la prochaine Semaine Sociale du Canada apporter sa précieuse contribution à cette réalisation progressive de la doctrine sociale de l'Eglise, pour le plus grand bien de votre chère patrie.

\section{SYNDICATS ET GROUPEMENTS PROFESSIONNELS DANS UNE DEMOCRATIE ORGANIQUE}

\section{Richard ARÈs, S.J.}

Il s'agit de situer syndicats et groupements professionnels dans une société démocratique sainement organisée. D'où une question préalable: pourquoi parler de démocratie, et de démocratie organique à propos des groupements syndicaux et professionnels?

\section{Pourquoi une démocratie organique?}

L'expression exprime tout d'abord un objectif global sur lequel tous les catholiques sociaux pourraient se mettre d'accord et à la réalisation duquel ils pourraient travailler de concert; ensuite un milieu vivant et sympathique au sein duquel syndicats et groupements professionnels pourraient non seulement se développer en liberté, mais encore assumer pleinement leurs responsabilités d'organes de la société.

L'un des besoins les plus profonds en même temps que l'un des espoirs les plus ardents de notre monde actuel, c'est le besoin et l'espoir d'une société vraiment humaine, d'hommes libres, à l'image et à la mesure de l'homme, respectueuse de sa dignité et de ses droits, dans l'organisation et le fonctionnement de laquelle l'homme aura son mot à dire et son rôle à jouer, et où il sera en mesure de mettre en échec les tyrannies, les dictatures et les monopoles de toutes sortes. Ce besoin et cet espoir ne se bornent pas à l'ordre politique, mais s'étendent en outre au terrain économique et social. Sur ce terrain aussi, il existe une immense aspiration à voir disparaître toutes les formes d'oppression et d'exploitation de l'homme, et à voir surgir une société ordonnée d'abord au service de l'homme et du peuple en général, une société organisée pour l'homme mais aussi par l'homme 